\title{
Non-existence of invariant circles
}

\author{
JOHN N. MATHER \\ Princeton University, Fine Hall, Princeton, NJ 08544, USA
}

(Received 17 June 1983)

Abstract. The dynamical system associated to the difference equation

$$
\Delta^{2} x_{n}=\frac{k}{2 \pi} \sin \left(2 \pi x_{n}\right)
$$

has been studied numerically by several authors. On the basis of numerical evidence, they conclude that there exists a number $k_{0} \approx 0.97$ such that there are homotopically non-trivial invariant circles for $|k| \leq k_{0}$ and there are none for $|k|>k_{0}$. In this note, we give a simple rigorous proof that there are none for $|k|>\frac{4}{3}$.

\section{Introduction}

We consider the space of mappings $\mathbb{Z} \rightarrow \mathbb{R}: n \mapsto x_{n}$. We are interested in those mappings which satisfy the equation $\Delta^{2} x_{n}=(k / 2 \pi) \sin \left(2 \pi x_{n}\right)$, where $\Delta^{2} x_{n}=$ $x_{n+1}-2 x_{n}+x_{n-1}$. The following is a standard way of expressing solutions of this difference equation as trajectories of a dynamical system. Let $y_{n}=x_{n}-x_{n-1}$. For solutions of the difference equation, we have

$$
\begin{aligned}
& y_{n+1}=x_{n+1}-x_{n} \\
& y_{n+1}-y_{n}=(k / 2 \pi) \sin \left(2 \pi x_{n}\right),
\end{aligned}
$$

which may be rewritten as

$$
\begin{aligned}
& x_{n+1}=x_{n}+y_{n}+(k / 2 \pi) \sin \left(2 \pi x_{n}\right) \\
& y_{n+1}=y_{n}+(k / 2 \pi) \sin \left(2 \pi x_{n}\right) .
\end{aligned}
$$

The question which we wish to study may be posed most simply if we think of the above equations as defining a mapping of the infinite cylinder. We let $(x, y)$ denote the coordinates for the infinite cylinder $T^{1} \times \mathbb{R}=(\mathbb{R} / \mathbb{Z}) \times \mathbb{R}$, where $x$ is defined modulo 1 . We let

$$
f_{k}: T^{1} \times \mathbb{R} \rightarrow T^{1} \times \mathbb{R}
$$

be the mapping defined by $f_{k}(x, y)=\left(x^{\prime}, y^{\prime}\right)$, where

$$
\begin{aligned}
& x^{\prime}=x+y+(k / 2 \pi) \sin (2 \pi x) \\
& y^{\prime}=y+(k / 2 \pi) \sin (2 \pi x) .
\end{aligned}
$$

By an invariant circle, we mean a subset $X \subset T^{1} \times \mathbb{R}$ such that $f_{k} X=X$ and $X$ is homeomorphic to $T^{1}=\mathbb{R} / \mathbb{Z}$. A subset $X \subset T^{1} \times \mathbb{R}$ which is homeomorphic to the circle is homotopically non-trivial if and only if its projection on $T^{1}$ is of degree \pm 1 . Intuitively, this means it goes once around the annulus. 
Here is our main result:

THEOREM. If $|k|>\frac{4}{3}$, then there are no homotopically non-trivial circles in $T^{1} \times \mathbb{R}$ which are invariant under $f_{k}$.

J. Greene told me about this problem, and I have had several conversations with him about it. He has done detailed numerical studies [7] of $f_{k}$ and found that there is a number $k_{0} \approx 0.97$ such that $f_{k}$ admits an invariant circle which is homotopically non-trivial when $|k| \leq k_{0}$ and there is none such when $|k|>k_{0}$. Several other authors have come to the same conclusion on the basis of numerical studies ([2], [5], [8], [12]). However, this conclusion has not yet been proved rigorously.

Our result is a very simple consequence of a theorem of $G$. D. Birkhoff $([4, \S 3]$ and $[3, \S 44]$ ). M. Herman has recently given a more detailed account of Birkhoff's result and related results [9].

Our method is the same as we used in [10]. However, because of the considerable recent interest in the mapping $f_{k}$, it seems to us worthwhile to publish our result.

\section{Birkhoff's theorem}

In this section we state the theorem of Birkhoff which we will use. We need the following definition, both for the statement of Birkhoff's theorem, and to extend Birkhoff's proof to the situation which we consider.

Definition. Consider a $C^{1}$ embedded curve $\gamma: J \rightarrow T^{1} \times \mathbb{P}$ where $J=(-\infty, a),(-\infty, a]$, or $(-\infty, \infty)$ and $a \in \mathbb{R}$. Suppose $\gamma(t)_{2} \rightarrow-\infty$, as $t \rightarrow-\infty$, where $(x, y)_{2}=y$, for $x \in T^{1}$, $y \in \mathbb{R}$. Let $\gamma^{\prime}: J \rightarrow \mathbb{R}^{2}$ denote the first derivative of $\gamma$. We define $\delta_{\gamma}: J \rightarrow \mathbb{R}$, called the deviation of $\gamma^{\prime}$ from the vertical, to be the unique continuous function which has the following two properties:

(a) $\delta_{\gamma}(t)$ is congruent $(\bmod 2 \pi)$ to the angle from the vertical vector $(\partial / \partial y)_{\gamma(t)}$ to $\gamma^{\prime}(t)$, where the counterclockwise direction is taken as the positive direction;

(b) given $t \in J$ such that $\gamma(s)_{2} \leq \gamma(t)_{2}$, for all $s \leq t$, we have $-\pi / 2 \leq \delta_{\gamma}(t) \leq \pi / 2$.

We will sketch a proof of the existence and uniqueness of $\delta_{\gamma}$ in $\S 3$, following a method suggested to us by $\mathrm{W}$. Thurston. Note that the existence and uniqueness of $\delta_{\gamma}$ depends on the fact that $\gamma$ is embedded, i.e. $\gamma$ is one-to-one and $\gamma^{\prime}$ never vanishes. It is not enough to assume that $\gamma$ is immersed (i.e. $\gamma^{\prime}$ never vanishes). We also need the following:

Definition. Let $g: T^{1} \times \mathbb{R} \rightarrow T^{1} \times \mathbb{R}$ be a $C^{1}$ diffeomorphism which maps each end of $T^{1} \times \mathbb{R}$ to itself. We will say that $g$ is tilted to the left (resp. right) if, for each vertical line $x=$ const. in $T^{1} \times \mathbb{R}$, its image under $g$ has everywhere positive (resp. negative) deviation from the vertical.

Example 2.1. Let $h: \mathbb{R} \rightarrow \mathbb{R}$ be a $C^{1}$ mapping of period 1. Let $g: T^{1} \times \mathbb{R} \rightarrow T^{1} \times \mathbb{R}$ be the mapping given by $g(x, y)=\left(x^{\prime}, y^{\prime}\right)$ where

$$
\begin{aligned}
& x^{\prime}=x+y+h(x) \\
& y^{\prime}=y+h(x)
\end{aligned}
$$

This mapping is tilted to the right. 
The mapping $f_{k}$ which we discussed in the introduction is a special case of this mapping $g$, corresponding to $h(x)=(k / 2 \pi) \sin (2 \pi x)$.

Birkhoff'S Theorem $\left([4, \S 3],[3, \S 44]\right.$, and [9, Chap. I]). Let $g: T^{1} \times \mathbb{R} \rightarrow T^{1} \times \mathbb{R}$ be a $C^{1}$ diffeomorphism. Suppose that $\mathrm{g}$ preserves the area form $d x d y$, maps each end of $T^{1} \times \mathbb{R}$ to itself, preserves orientation, and is tilted (either to the left or to the right). Let $U$ be an open subset of $T^{1} \times \mathbb{R}$ such that $\mathrm{g} U=U, U$ is homeomorphic to $T^{1} \times \mathbb{R}$, and $T^{1} \times(-\infty, a] \subset U \subset T^{1} \times(-\infty, b)$, for some $a<b, a, b \in \mathbb{R}$. Then the frontier of $U$ in $T^{1} \times \mathbb{R}$ is the graph of a Lipschitz function $\mu: T^{1} \rightarrow \mathbb{R}$, i.e. $\bar{U}-U=\left\{(x, \mu(x)): x \in T^{1}\right\}$. Example 2.2. The mapping given by (1) satisfies all the conditions we imposed on $g$ in the above theorem.

Example 2.3. Let $X \subset T^{1} \times \mathbb{R}$ be a homotopically non-trivial circle. One of the components of the complement of $X$ in $T^{1} \times \mathbb{R}$ satisfies the conditions we imposed on $U$ in the above theorem. This is a classical result in topology: it follows immediately from the Schoenflies theorem. (Embed $T^{1} \times \mathbb{R}$ as the annulus in the plane.)

Remark. From the last example, we obtain a corollary of Birkhoff's theorem: if $X$ is a g-invariant, homotopically non-trivial circle in $T^{1} \times \mathbb{R}$, then $X$ is the graph of a Lipschitz function $\mu: T^{1} \rightarrow \mathbb{R}$.

There is a numerical invariant, called the flux, associated to a mapping $g$ which satisfies the conditions which we stated in the above theorem. When this invariant is non-zero, Birkhoff's theorem is vacuously true.

Definition. Let $g$ satisfy the conditions in Birkhoff's theorem. The flux $C(g)$ is defined to be

$$
\text { Area }(g U \backslash U) \text { - Area }(U \backslash g U) \text {, }
$$

where $U$ is any open set in $T^{1} \times \mathbb{R}$ such that $T^{1} \times(-\infty, a] \subset U \subset T^{1} \times(-\infty, b)$, for some $a<b, a, b \in \mathbb{R}$.

From the fact that $g$ is an area preserving homeomorphism of $T^{1} \times \mathbb{R}$ which maps each end to itself, it follows that $C(g)$ is independent of $U$. Clearly, if there exists $U$ which satisfies the conditions which we imposed in our statement of Birkhoff's theorem, then we may use it to compute $C(g)$, and we obtain $C(g)=0$. In other words, if the flux is non-zero, there is no $U$ which satisfies the conditions imposed in Birkhoff's theorem. In this case, Birkhoff's theorem is vacuously true.

Example 2.4. Let $g$ be the mapping given by (1). Then

$$
C(g)=\int_{0}^{1} h(x) d x .
$$

This may be seen by taking the set $U$ used in the definition of $C(g)$ to be $T^{1} \times(-\infty, 0)$. In particular, $C\left(f_{k}\right)=0$, where $f_{k}$ is the mapping defined in the introduction.

The hypotheses of the theorems in $[4, \S 3]$ and $[3, \S 44]$ are slightly different from those in our version of Birkhoff's theorem. In fact, our version is not a consequence of either of these results, but it may be proved in a similar way. A detailed proof 
of a version of Birkhoff's theorem which is very similar to ours is given in [9]. In $\S 4$, we give a brief outline of Birkhoff's proof.

\section{Existence and uniqueness of $\delta_{\gamma}$}

We assume that $\gamma$ satisfies the conditions stated in the definition in $\S 2$. In view of the fact that $\gamma(t)_{2} \rightarrow-\infty$ as $t \rightarrow-\infty$, we have that there exists $t_{0} \in J$ such that $\gamma(s)_{2} \leq \gamma\left(t_{0}\right)_{2}$, for $s \leq t_{0}$. Obviously, there is a unique continuous $\delta_{\gamma}$ satisfying (b) for $t=t_{0}$ as well as (a) for all $t$. It is enough to show that given $t_{1} \in J$ such that $\gamma(s)_{2} \leq \gamma\left(t_{1}\right)_{2}$, for all $s \leq t_{1}$, we have $-\pi / 2 \leq \delta_{\gamma}\left(t_{1}\right) \leq \pi / 2$.

The proof of this was suggested to me by $W$. Thurston. It is crucial to use the fact that $\gamma$ is embedded. For simplicity, we will suppose $t_{0}<t_{1}$; the other case is treated similarly.

Step 1. We reduce to the case when $\gamma$ is vertical in a neighbourhood of $t_{0}$ and $t_{1}$ and $\gamma(s)_{2} \leq \gamma\left(t_{i}\right)_{2}$ for $i=1,2$. This may be done by deforming $\gamma$ in an arbitrarily small neighbourhood of $t_{0}$ and $t_{1}$. In this case, what we must prove reduces to $\delta_{\gamma}\left(t_{1}\right)=0$.

Step 2. Since $\gamma$ is vertical in a neighbourhood of $t_{0}$ and $\gamma(s)_{2} \leq \gamma\left(t_{0}\right)_{2}$ for $s \leq t_{0}$, we may isotope $T^{1} \times \mathbb{R}$, leaving $T^{1} \times\left[\gamma\left(t_{0}\right)_{2}-\varepsilon, \infty\right)$ pointwise fixed, so as to deform $\gamma$ into a curve satisfying the condition that $\gamma \mid\left(-\infty, t_{0}+\varepsilon\right]$ be vertical. Here, $\varepsilon$ is a small positive number. The fact that this may be done is well known to differential topologists and intuitively obvious. Obviously, we have $\delta_{\gamma}\left(t_{1}\right)=0$ for the deformed $\gamma$ if and only if we have it for the original $\gamma$.

Step 3. Now $\gamma$ is vertical on $\left(-\infty, t_{0}+\varepsilon\right]$ and in a neighbourhood of $t_{1}$, and $\gamma(s)_{2} \leq \gamma\left(t_{1}\right)_{2}$ for $s \leq t_{1}$. Then

$$
\gamma\left(t_{0}\right)_{2}<\gamma\left(t_{0}+\varepsilon\right)_{2} \leq \gamma\left(t_{1}\right)_{2}
$$

We may find an isotopy of $T^{1} \times \mathbb{R}$ which preserves the $y$-coordinate, which is a rotation of constant speed in the $x$ coordinate on $T^{1} \times\left[\gamma\left(t_{1}\right)_{2}-\varepsilon, \infty\right)$, which is the identity on $T^{1} \times\left(-\infty, \gamma\left(t_{0}\right)_{2}+\varepsilon\right]$, and such that at the end of the isotopy $\gamma\left(t_{0}\right)_{1}=$ $\gamma\left(t_{1}\right)_{1}$. Obviously, we have $\delta_{\gamma}\left(t_{1}\right)=0$ for the deformed $\gamma$ if and only if we have it for the original $\gamma$.

Step 4. Now $\gamma$ is vertical on $\left(-\infty, t_{0}+\varepsilon\right]$ and in a neighbourhood of $t_{1}$, we have $\gamma(s)_{2} \leq \gamma\left(t_{1}\right)_{2}$ for $s \leq t_{1}$, and $\gamma\left(t_{0}\right)_{1}=\gamma\left(t_{1}\right)_{1}$. There exist circles $C_{1}, C_{2}$ on $T^{1} \times \mathbb{R}$ such that

$$
\begin{aligned}
& C_{1} \cap \text { image } \gamma=\gamma\left(t_{0}+\varepsilon\right), \\
& C_{2} \cap \text { image } \gamma=\gamma\left(t_{1}-\varepsilon\right),
\end{aligned}
$$

and

$$
C_{1} \cap C_{2}=\varnothing \text {. }
$$

We may find an isotopy of $T^{l} \times \mathbb{R}$ which is the identity outside the annulus bounded by $C_{1}$ and $C_{2}$ such that at the end of the isotopy $\gamma$ is vertical on $\left(-\infty, t_{1}+\varepsilon\right]$. Again, the fact that this may be done is well known to differential topologists and intuitively obvious. Obviously, the deformed $\gamma$ satisfies $\delta_{y}\left(t_{1}\right)=0$ and this implies the same equality for the original $\gamma$. 


\section{Proof of Birkhoff's theorem}

We give a brief sketch, following Birkhoff's ideas closely. We say a point $x$ in $U$ is positively (resp. negatively) accessible if there is an embedded curve $\gamma:(-\infty, a] \rightarrow U$ satisfying $\gamma(t)_{2} \rightarrow-\infty$, as $t \rightarrow-\infty$, and having positive (resp. negative) deviation from the vertical, such that $\gamma(a)=x$. We let $W_{+}$(resp. $W_{-}$) denote the set of points in $U$ which are positively (resp. negatively) accessible.

We will prove Birkhoff's theorem in the case that $g$ is tilted to the right. Obviously, the case when $g$ is tilted to the left can be reduced to this case, by replacing $g$ by $g^{-1}$.

In the case $g$ is tilted to the right, it is easily checked that $g\left(\bar{W}_{-} \cap U\right) \subset W_{-}$and $g^{-1}\left(\bar{W}_{+} \cap U\right) \subset W_{+}$. From this, it follows that $W_{-}=U=W_{+}$, in view of the following facts: $g$ is area preserving and there exist $a<b$ such that

$$
T^{1} \times(-\infty, a] \subset W_{-} \cap U \cap W_{+} \subset W_{-} \cup U \cup W_{+} \subset T^{1} \times(-\infty, b] .
$$

From the fact that $W_{-}=U=W_{+}$, it follows that for each $x \in T^{1}$, there exists $a_{x} \in \mathbb{R}$ such that

$$
U \cap(x \times \mathbb{R})=x \times\left(-\infty, a_{x}\right) .
$$

The proof of this is similar to the proof in $\S 3$; it is mentioned in Birkhoff's papers and also in Herman's notes [9].

If $u: \mathbb{R} \rightarrow \mathbb{R}$ is a $C^{1}$ function we let $\phi_{u}: T^{1} \times \mathbb{R} \rightarrow T^{1} \times \mathbb{R}$ be the diffeomorphism

$$
\phi_{u}(x, y)=(x+u(y), y) \text {. }
$$

Let $g_{u}=\phi_{u} g \phi_{u}^{-1}$. Then $\phi_{u} U$ is invariant for $g_{u}$. If $u$ is sufficiently close to 0 in the Whitney $C^{1}$ topology, then $g_{u}$ is tilted to the same side as $g$. Hence $\phi_{u} U$ satisfies the condition that for each $x \in T^{1}$, there exists $a_{u x} \in \mathbb{R}$ such that

$$
\phi_{u} U \cap(x \times \mathbb{R})=x \times\left(-\infty, a_{u x}\right) .
$$

Since this is true for any $u$ in a $C^{1}$ sufficiently small neighbourhood of 0 , it follows that the frontier of $U$ is the graph of a Lipschitz function, as asserted in Birkhoff's theorem.

\section{The generating function}

Let $g$ be the mapping of example 2.1. By example 2.4, the flux $C(g)$ is $\int_{0}^{1} h(x) d x$. Since Birkhoff's theorem is vacuously true when $C(g) \neq 0$, we will suppose that $\int_{0}^{1} h(x) d x=0$. We let $H$ be an indefinite integral of $h$. Since $\int_{0}^{1} h(x) d x=0$, we have that $H$ is periodic of period 1 .

What is known in classical mechanics as the generating function for $g$ is defined by

$$
G\left(x, x^{\prime}\right)=-\frac{\left(x^{\prime}-x\right)^{2}}{2}-H(x)
$$

The equation (1) of example (2.1) is equivalent to

$$
\begin{aligned}
& y=\frac{\partial G\left(x, x^{\prime}\right)}{\partial x} \\
& y^{\prime}=-\frac{\partial G\left(x, x^{\prime}\right)}{\partial x^{\prime}}
\end{aligned}
$$


Now suppose $g$ admits a homotopically non-trivial circle $X$. In view of Birkhoff's theorem and the remark following example 2.3, $X$ is the graph of a Lipschitz function $\mu: T^{\prime} \rightarrow \mathbb{R}$. Therefore, there exists a homeomorphism $g_{0}: T^{1} \rightarrow \mathbb{R}$ such that

$$
g(x, \mu(x))=\left(g_{0}(x), \mu\left(g_{0}(x)\right)\right) .
$$

Since $g$ maps each end of $T^{1} \times \mathbb{R}$ to itself, it maps each complementary component of $X$ into itself. Moreover, $g$ is orientation preserving, so $g_{0}$ is orientation preserving. From equation (2), we get

$$
\frac{\partial}{\partial x}\left[G(\bar{x}, x)+G\left(x, x^{\prime}\right)\right]=0,
$$

where $\bar{x}=g_{0}^{-1}(x), x^{\prime}=g_{0}(x)$.

\section{Proof of the theorem}

We continue with the notations of the previous section. Since $g_{0}=\mu_{0}^{-1} g \mu_{0}$, where $\mu_{0}=(1, \mu): T^{1} \rightarrow$ graph $\mu$, and $\mu_{0}$ and $g$ are Lipschitz, it follows that $g_{0}$ is Lipschitz. Likewise $g_{0}^{-1}=\mu_{0}^{-1} g^{-1} \mu_{0}$ is Lipschitz. Obviously, a Lipschitz function is absolutely continuous, so we may apply the classical theorem in Lebesgue theory which asserts that an absolutely continuous function is differentiable almost everywhere and the indefinite integral of its derivative is the original function (up to a constant). This theorem is stated and proved in $[13,11.7]$.

Hence, we may differentiate (3), obtaining

$$
\frac{d g_{0}^{-1}(x)}{d x}+\frac{d g_{0}(x)}{d x}=2+h^{\prime}(x)
$$

where $h^{\prime}$ is the derivative of $h$. Equation (4) is valid wherever $d g_{0}(x) / d x$ and $d g_{0}^{-1}(x) / d x$ are defined. So, it is valid almost everywhere.

Let $L$ be the larger of the Lipschitz constants of $g_{0}$ and $g_{0}^{-1}$, i.e.

$$
L=\max \left\{\sup \frac{\left|g_{0}(x)-g_{0}\left(x^{\prime}\right)\right|}{\left|x-x^{\prime}\right|}, \sup \frac{\left|g_{0}^{-1}(x)-g_{0}^{-1}\left(x^{\prime}\right)\right|}{\left|x-x^{\prime}\right|}\right\} .
$$

We have that $d g_{0}(x) / d x$ and $d g_{0}^{-1}(x) / d x$ are non-negative, since $g_{0}$ and $g_{0}^{-1}$ are orientation preserving. In view of the definition of $L$, it then follows that

$$
\begin{aligned}
& L^{-1} \leq \frac{d g_{0}(x)}{d x} \leq L, \\
& L^{-1} \leq \frac{d g_{0}^{-1}(x)}{d x} \leq L .
\end{aligned}
$$

Let $m=\min h^{\prime}, M=\max h^{\prime}$. From (4) and (5), we get

$$
2 L^{-1} \leq 2+m
$$

In particular,

$$
m>-2
$$


Since (7) was derived under the sole assumption that $g$ admits a homotopically non-trivial invariant circle, it is a necessary condition for the existence of a homotopically non-trivial invariant circle. We may obtain another necessary condition, as follows.

From the definition of $L$ and the theory of $[13,11.7]$, we get

$$
L=\max \left\{\text { ess.sup. } \frac{d g_{0}(x)}{d x}, \text { ess.sup. } \frac{d g_{0}^{-1}(x)}{d x}\right\} .
$$

Here ess.sup. means 'essential supremum', in the sense of measure theory. Since $d g_{0}(x) / d x, d g_{0}^{-1}(x) / d x$ are derivatives of Lipschitz functions, the essential supremum of each of them is the same as the supremum over the set where it is defined.

From (4), (5), and (8), we get

$$
L^{-1}+L \leq 2+M
$$

Since the function $L^{-1}+L$ is a monotone increasing function for $L \geq 1,(6)$ and (9) give

$$
\frac{2+m}{2}+\frac{2}{2+m} \leq 2+M .
$$

Here we are using the fact that $m \leq 0$, since $h$ is periodic.

In the case $g=f_{k}$, we have $h(x)=(k / 2 \pi) \sin (2 \pi x)$, so $M=|k|, m=-|k|$. Inequalities (7) and (10) imply

$$
|k| \leq \frac{4}{3}
$$

Since (11) was derived under the assumption of the existence of a homotopically non-trivial circle, this finishes the proof.

\section{Addition remarks}

(1) The above text is the preprint [11] with only minor changes. Since I wrote it, Fathi [6] has given another detailed proof of Herman's version [9] of Birkhoff's theorem.

(2) Aubry, Le Daeron, and André have proved a necessary criterion for the existence of invariant circles [1]. It appears that their result together with, perhaps lengthy, numerical calculations imply the main result of this paper.

(3) Let $g$ be a mapping of the type given in example 2.1. Equation (3) is equivalent to

$$
g_{0}^{-1}(x)+g_{0}(x)=2 x+h(x) .
$$

Conversely, if there is a mapping $g_{0}: \mathbb{R} \rightarrow \mathbb{R}$ which is strictly increasing and satisfies $g_{0}(x+1)=g_{0}(x)+1$ and the above equation, then $g$ has a homotopically non-trivial invariant circle, which is the graph of the function

$$
\mu(x)=G_{1}\left(x, g_{0}(x)\right)
$$

where $G_{1}=\partial G\left(x, x^{\prime}\right) / \partial x$. This observation is due to $M$. Herman who used it in his lectures in Brazil in the summer of 1981 to construct a remarkable example where $g$ is of class $C^{3-\varepsilon}$ and has a non transitive invariant circle with irrational rotation number. (See [9, chap. III]). 
(4) The proof given above shows that if $g$ has a homotopically non-trivial invariant circle, then (7) and (10) are satisfied. In my graduate course in the spring of 1983, I raised the question of whether the hypothesis that $\boldsymbol{g}$ has a homotopically non-trivial invariant circle implies any further conditions on $m$ and $M$. Rafael Llave answered this question: he showed that for any $m \leq 0$ and any $M \geq 0$ satisfying (7) and (10), there exists $g$ which has a homotopically non-trivial invariant circle and which satisfies $m=\min h, M=\max h$. His proof is given in the appendix.

(5) The referee has pointed out that the inequalities of $\S 6$ are contained in [9, chaps. II and III] and that the example of the appendix is contained in [9, chap. III. 9], where it was used to settle a different question. He comments that these inequalities and examples were discussed by $M$. Herman in various lectures given since 1980 in Palaiseau, Zurich, Rio, ....

\section{APPENDIX}

\section{R. Llave}

THeOREM. Let $m<0<M$ be two real numbers satisfying (7) and (10). Then there exist two $C^{\infty}$ functions $g_{0}, h: \mathbb{R} \rightarrow \mathbb{R}$ such that $g_{0}$ is increasing, $g_{0}(x+1)=g_{0}(x)+1$,

$$
g_{0}^{-1}(x)+g_{0}(x)=2 x+h(x),
$$

and

$$
\max h^{\prime}(x)=M, \quad \min h^{\prime}(x)=m
$$

Proof. Since $-2<m<0$, there exists $l$ such that

$$
\frac{2}{m+2} l+\frac{m+2}{2}(1-l)=1
$$

Let $g_{0}$ be the piecewise linear function defined in $[0,1]$ satisfying

$$
\begin{array}{ll}
g_{0}(0)=0 & \\
g_{0}^{\prime}(x)=2 /(2+m), & x \in[0, l / 2] \cup(1-l / 2,1] ; \\
g_{0}^{\prime}(x)=(2+m) / 2, & x \in(l / 2,1-l / 2) .
\end{array}
$$

Then $g_{0}(1)=1$, so $g_{0}$ can be extended to all of $\mathbb{R}$ so as to satisfy $g_{0}(x+1)=g_{0}(x)+1$. Let $h$ be defined by (A1). Then

$$
m=\min h^{\prime}(x), \quad M_{1}=\max h^{\prime}(x),
$$

where $M_{1}$ is defined by the equation

$$
2 /(2+m)+(2+m) / 2=2+M_{1} .
$$

See figure 1 .

We can round the corners of $g_{0}$ so as to get infinitely differentiable functions $g_{0}$ and $h$ (again defined by (A1)) such that (A3) holds. Finally, by altering $g_{0}$ in an arbitrarily small interval, so as to increase its derivative there, we can arrange that $\max h^{\prime}(x)=M$ and all the other conditions of the theorem hold as well, since $M \geq M_{1}$, by $(10)$. 


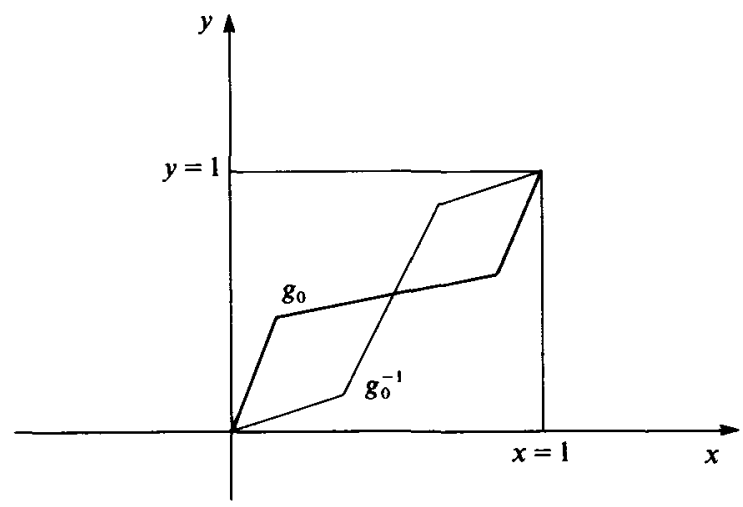

Figure 1

\section{REFERENCES}

[1] Aubry, Le Daeron, \& André. Classical ground states of a one-dimensional model for incommensurate structures. Preprint (1982).

[2] G. Benettin, L. Galgani \& J. Strelcyn. Kolmogorov entropy and numerical experiments. Phys. Rev. A 14 (1976), p. 2338.

[3] G. D. Birkhoff. Surface transformrations and their dynamical applications. Acta Math. 43 (1922), 1-119. Reprinted in Collected Mathematical Papers, Vol. II, Amer. Math. Soc.: New York (1950), pp. 111-229.

[4] G. D. Birkhoff. Sur quelques courbes fermées remarquables. Bull. Soc. Math. de France 60 (1932), 1-26. Reprinted in Collected Mathematical Papers, Vol. II, Amer. Math. Soc.: New York (1950), pp. 418-443.

[5] B. V. Chirikov. A universal instability of many-dimensional oscillator systems. Physics Reports $\mathbf{5 2}$ (1979), 263-379.

[6] A. Fathi. Une interprétation plus topologique de la démonstration du théorème de Birkhoff. Appendix of [9].

[7] J. M. Greene. A method for determining a stochastic transition. J. Math. Phys. 20 (1979), 1183-1201.

[8] R. H. G. Hellemann \& T. Bountis. Periodic solutions of arbitrary period, variational methods. In Stochastic Behaviour in Classical and Quantal Systems, (ed. G. Casati and J. Ford). Springer Verlag: New York (1979), p. 353.

[9] M. Herman. Introduction à l'étude des courbes invariantes par les diffeomorphismes de l'anneau. Asterisque 103-104 (1983).

[10] J. Mather. Glancing billiards. Ergod. Th. \& Dyn. Sys. 2 (1982), 397-403.

[11] J. Mather. Non-existence of invariant circles. Preprint (1982).

[12] G. E. Powell \& J. C. Percival. A spectral entropy method for distinguishing regular and irregular motion of Hamiltonian systems. J. Phys. A 12 (1979), p. 2053-2071.

[13] E. C. Titchmarsh. The Theory of Functions, (2nd edition). Oxford Univ. Press: Oxford (1939). 